\title{
EFFICIENT CONTRAST ENHANCEMENT USING GAMMA CORRECTION WITH MULTILEVEL THRESHOLDING AND PROBABILITY BASED ENTROPY
}

\author{
S.Gayathri ${ }^{1}$, N.Mohanapriya ${ }^{2}$, B.Kalaavathi ${ }^{3}$ \\ ${ }^{1} P G$ student, Computer Science and Engineering, Vivekanandha College of Engineering for Women, Tamilnadu, India \\ ${ }^{2}$ Assistant Professor, Computer Science and Engineering, Vivekanandha College of Engineering for Women, \\ Tamilnadu, India \\ ${ }^{3}$ Professor, Computer Science and Engineering, K.S.R institute of Engineering and technology, Tamilnadu, India
}

\begin{abstract}
In digital image enhancement, contrast enhancement plays an important role. The main objective of contrast enhancement is to correct the local contrast in different area of the image. So that the feature unseen in the dark or bright area is brought out and exposed to the human views. In this paper improve the brightness of the dimmed images and preservation of image feature via multilevel thresholding, gamma correction and probability based entropy. The proposed method preserves the feature of the image and brightness and also improves image quality.
\end{abstract}

Keywords: Contrast Enhancement, Gamma correction, Entropy, Multilevel thresholding. $* * *$

\section{INTRODUCTION}

The enhancement techniques for dimmed images can be categories into two methods, first one is direct enhancement method and another one is indirect enhancement methods [1]. This method applied for various applications such as general images, indoor and outdoor images. Contrast enhancement plays an important role in image enhancement. Poor contrast in digital images can be produced from many conditions, including lack of operator knowledge and inadequate of the image capture device [2].

Poor environmental conditions in the captured scene, such as the occurrence of clouds, lack of sunlight or indoor lighting, and some other condition. That also leads to reduced contrast quality of the images. The overall luminance is insufficient, and then the details of the image or video facial appearance will be buried. The main intention of contrast enhancement is to correct the local contrast in different area of the image. So that the feature hidden in the dark or bright area is brought out and exposed to the human views.

\section{RELATED WORK}

Contrast enhancement is to correct the local contrast in different area of the image. So that the feature out of sight in the dark or bright area is brought out and exposed to the human views. [12]. several contrast enhancement techniques are available, some of the techniques are discussed.

\subsection{Contextual and Variational Contrast}

\section{Enhancement}

Enhancement of the image done by using inter pixel of an image. The algorithm use 2-D histogram of input image that is created using association between the each pixel of input image and its neighboring pixel [3].

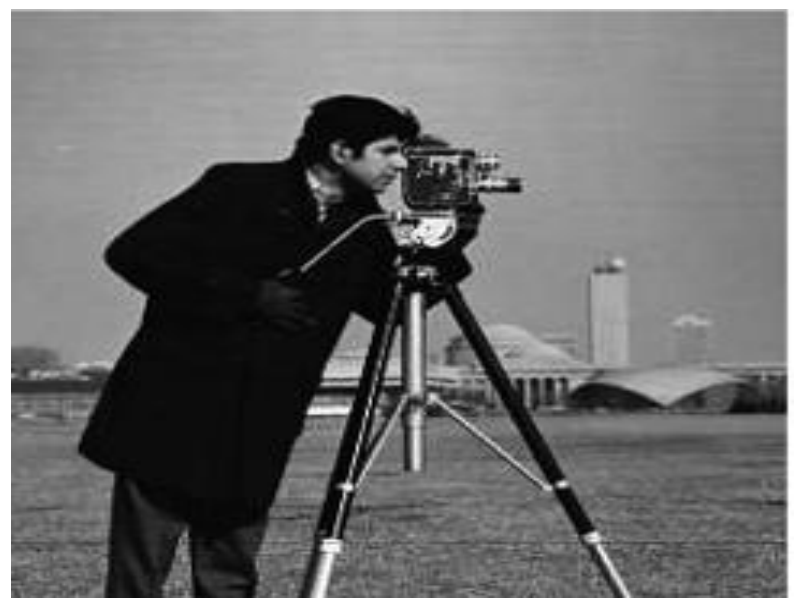

Fig -1: Input image

The smooth 2-D intention histogram is obtained by optimize the sum of frobenius norms of difference between the input image histogram and evenly distributed histogram. By increase the dynamic range low contrasts are improved.

High contrast also increased but not that much. This algorithm improves visual quality well but it required high computational time. 


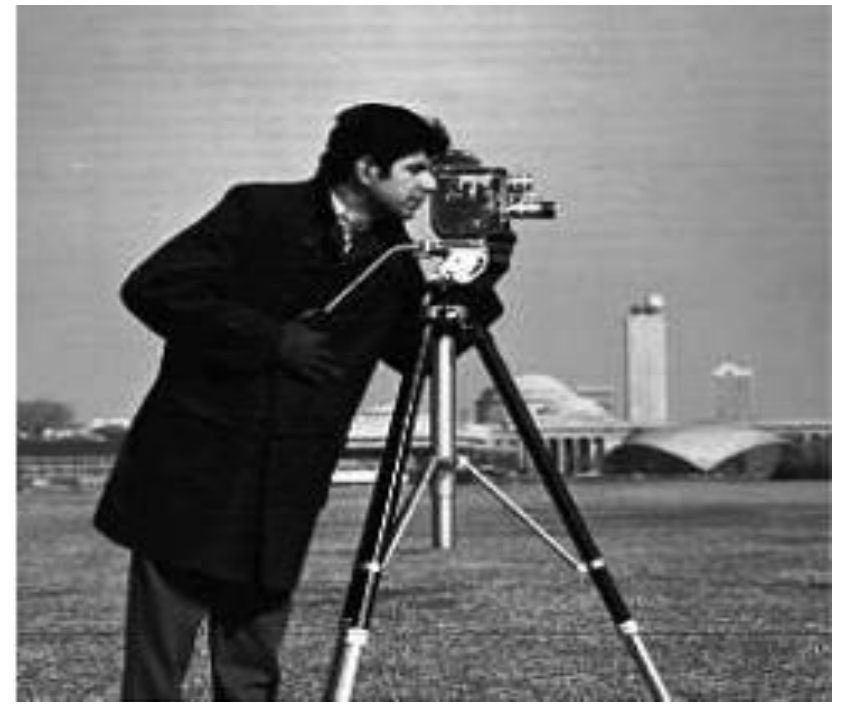

Fig -2: Enhanced image

\subsection{Recursively Separated and Weighted Histogram}

\section{Equalization for Brightness Preservation and}

\section{Contrast Enhancement}

These approaches mainly concentrate on preservation of brightness of an image and contrast of image [9]. The essential process done in this approach is to segment input images into two are additional sub- histograms recursively and adjust the sub- histograms with the help of weighting process. It is efficiently solve the problem of mean shift.

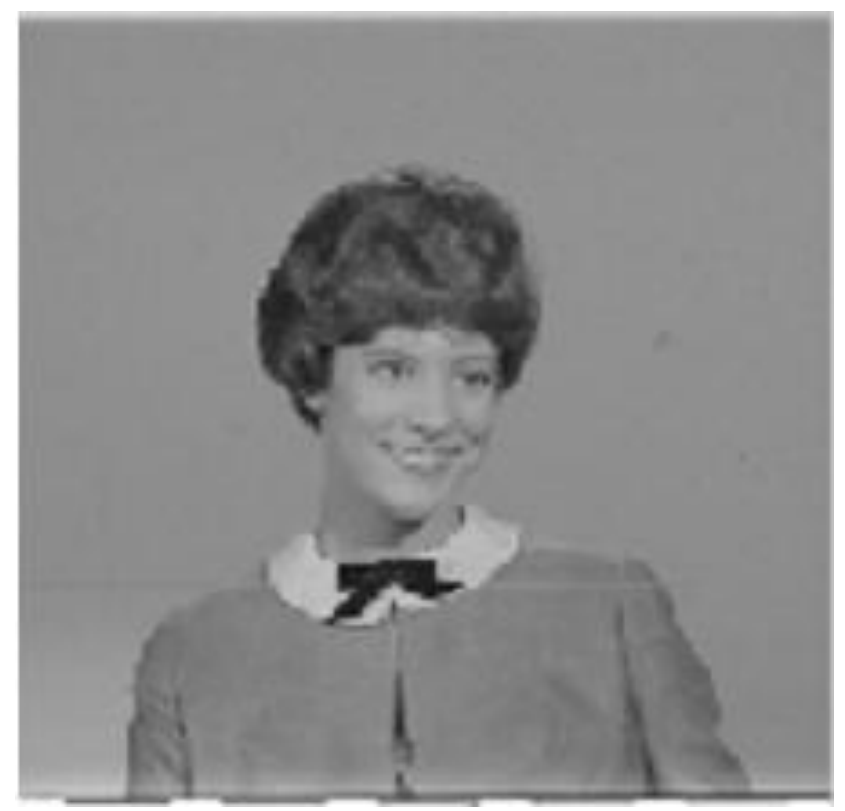

Fig -3: Original image

By adjust the sub-histograms of an image it includes multiequalization and preservation of brightness [8]. Some statistical value may lose while modifying the subhistograms.

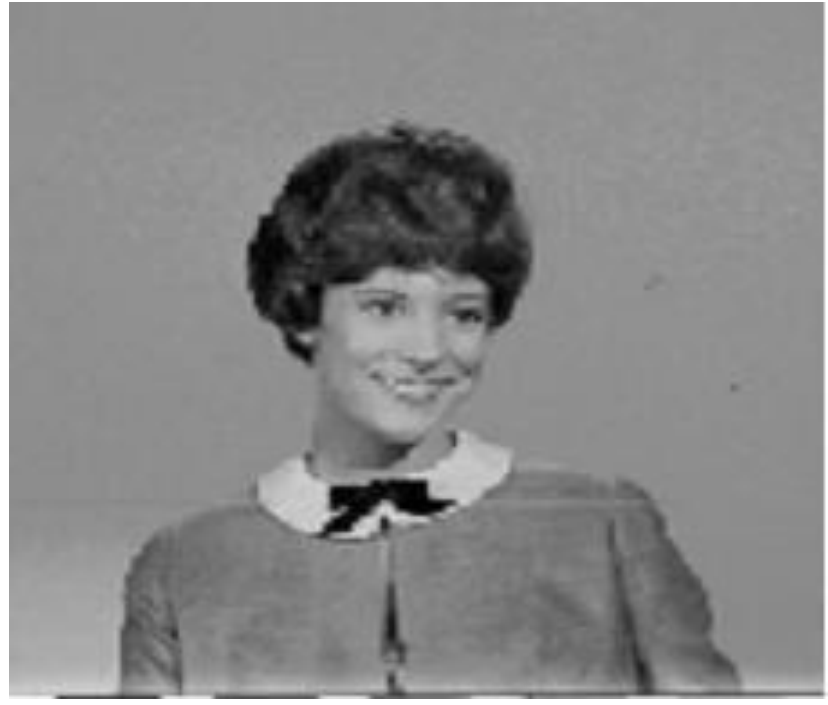

Fig -4: Output image of RSWHE

\subsection{Image Enhancement Based On Equal Area}

\section{Dualistic Sub-Image Histogram Equalization}

\section{Method}

This approach enhances the information efficiently and also maintains the luminance of an original image [11]. The basic process done in this approach is to decompose the input image into two equal sub-images according to the probability density function of the original image.

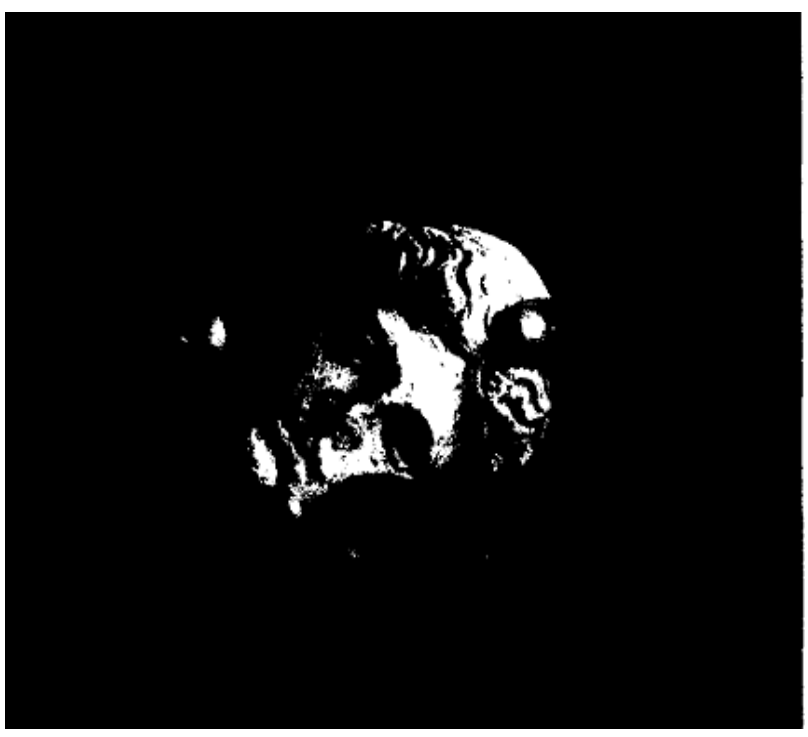

Fig -5: Original image

And two sub images are equalizing respectively. Finally, output image produced by combined the two sub-images into one. The outputs produced by this method maintain the original brightness of an input image and enhances the information effectively. If the image has high intensity distribution than cannot solve over equalization effect efficiently 


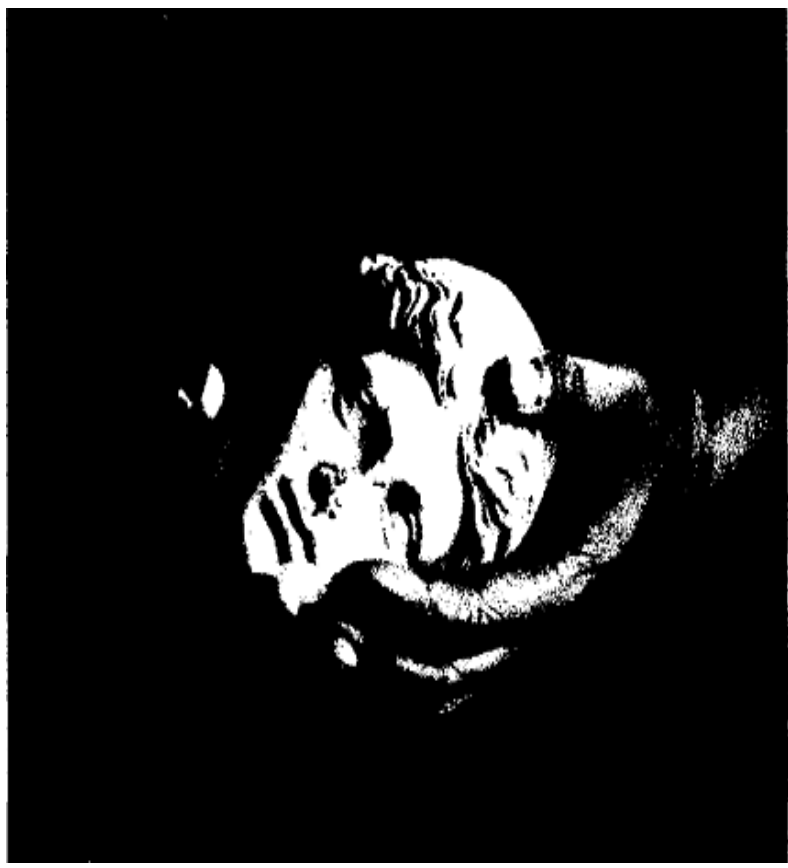

Fig -6: Enhanced image

\subsection{Recursive Sub-Image Histogram Equalization}

\section{Applied to Gray Scale Images}

This approach is the more reliable, because of the recursive environment and scalable preservation of brightness [8]. The basic process done in this approach is, instead of separating image only one time, it recursively separate the image at several time to get multiple sub-histograms.

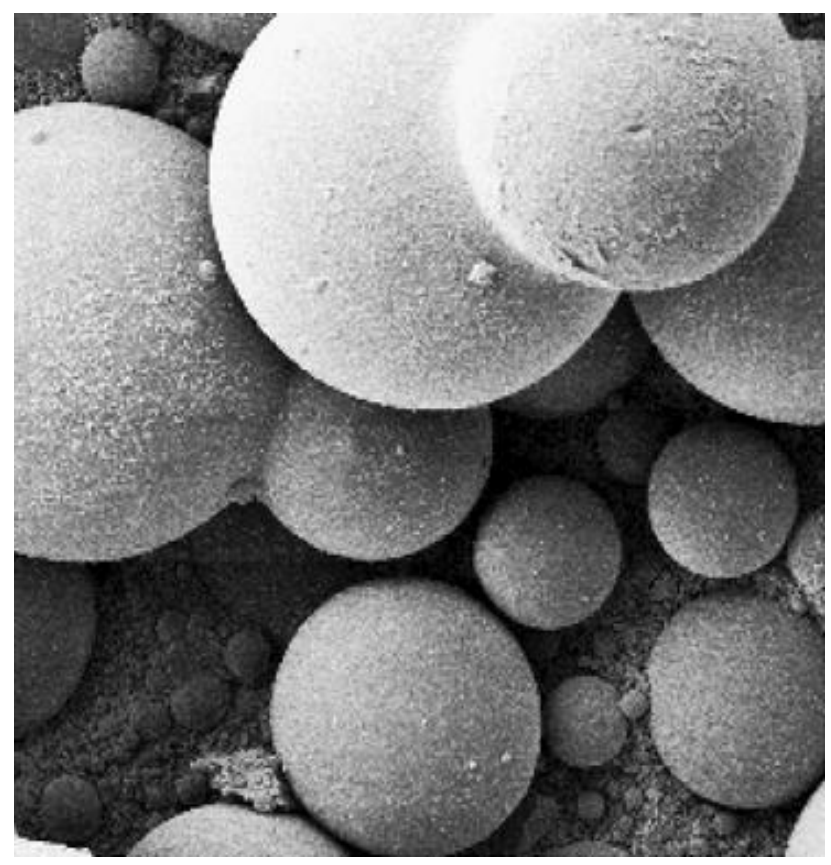

Fig.-7. Original image

This gives the advantage of scalable brightness preservation. But more sub-images consume more computational time.

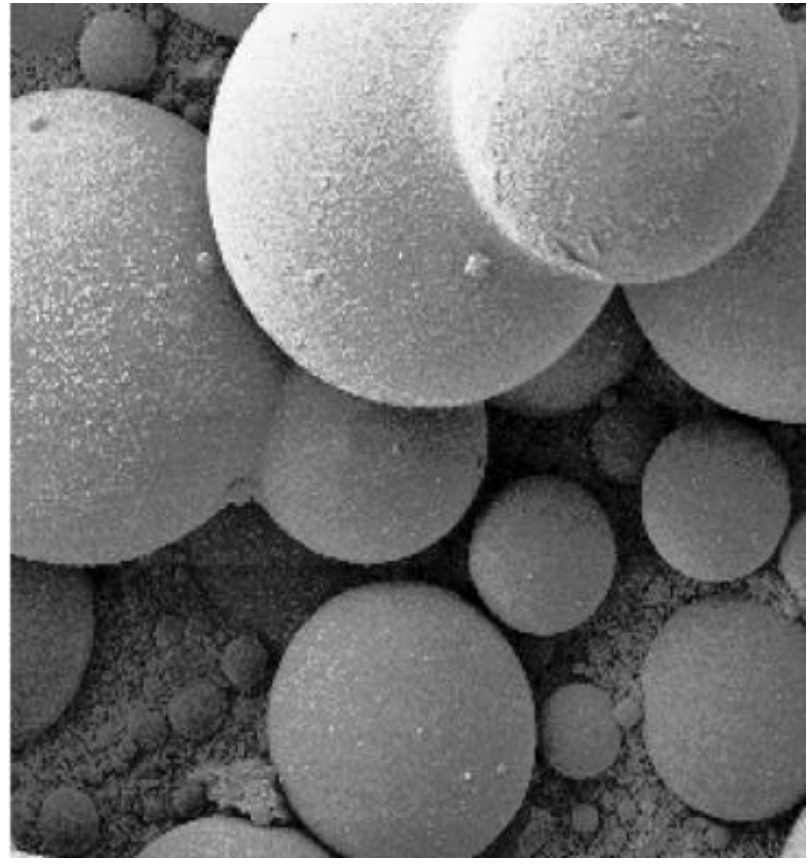

Fig.-8. Output of DSIHE

\subsection{Brightness Preserving Histogram Equalization} with Maximum Entropy: A Variational Perspective

These approaches enhance the image efficiently and also preserve the original brightness of the image. The basic idea of this approach, maximize the entropy by using target histogram [6].

That the mean brightness is fixed based on the constraints, than convert the original histogram to the target histogram by using histogram specification [10]. This approach does not exploit the discrete entropy.

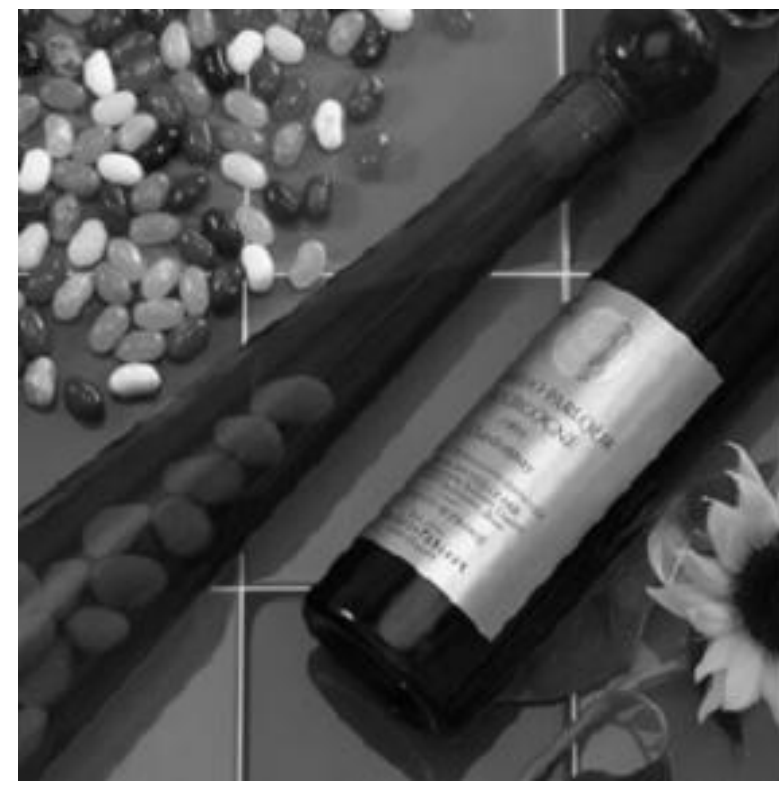

Fig.-9. Input image 


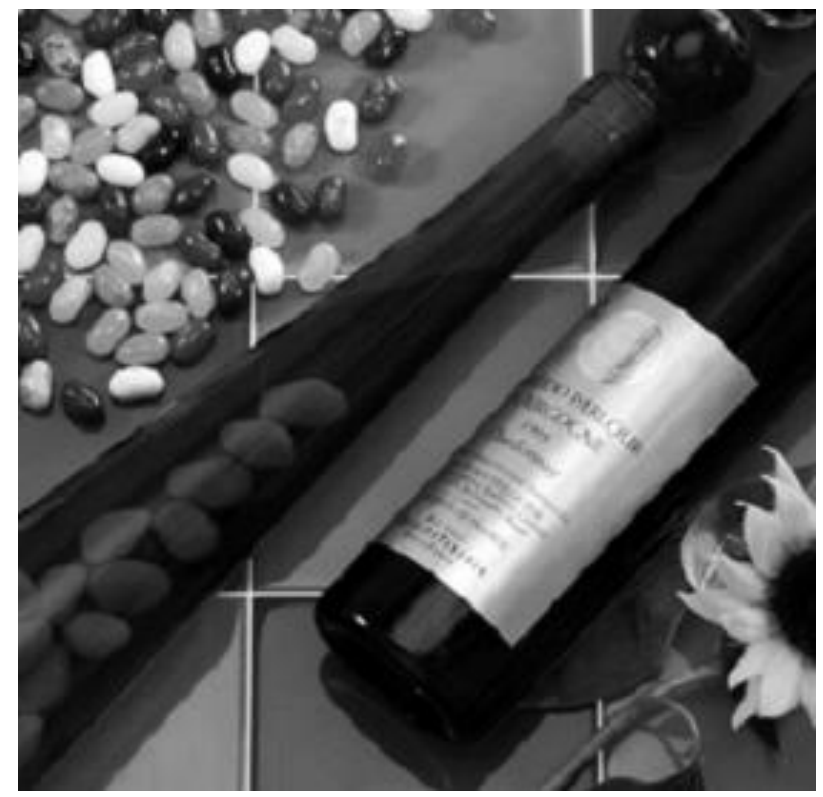

Fig.-10. Enhanced image

\subsection{Contrast Enhancement Using Brightness}

\section{Preserving Bi-Histogram Equalization}

The goal of this approach is to preservation of the mean brightness of an image at the time of enhance the contrast of given image [7]. The basic idea of this approach, utilize the autonomous histogram equalization independently. Two sub-image are produced by decompose the input image .The decomposition is done based on the mean.

It preserves the mean brightness of input image, it consume more computational time.

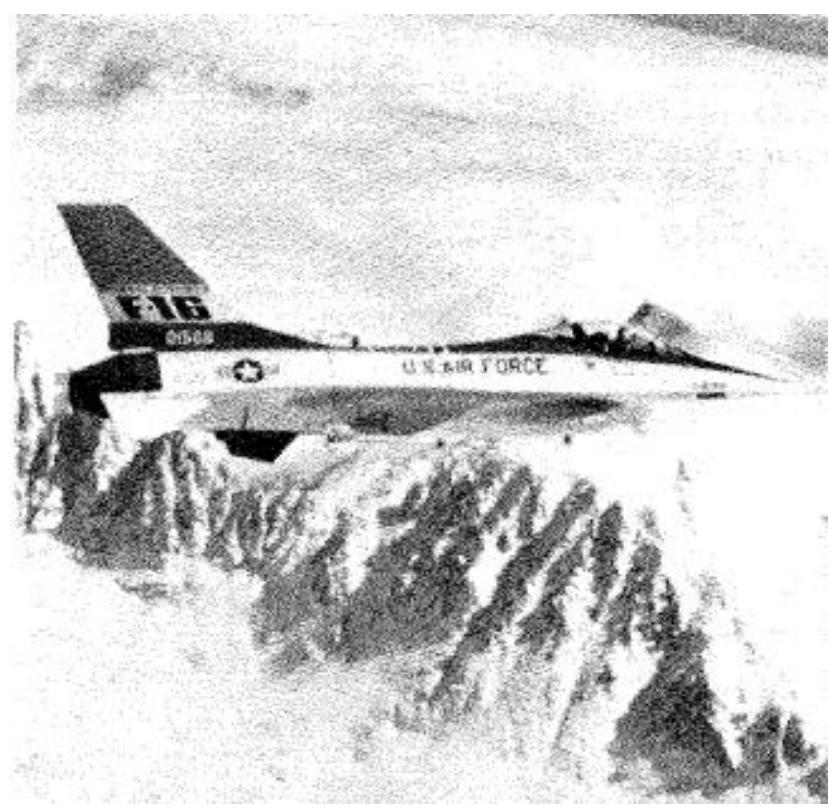

Fig.-11. Original image

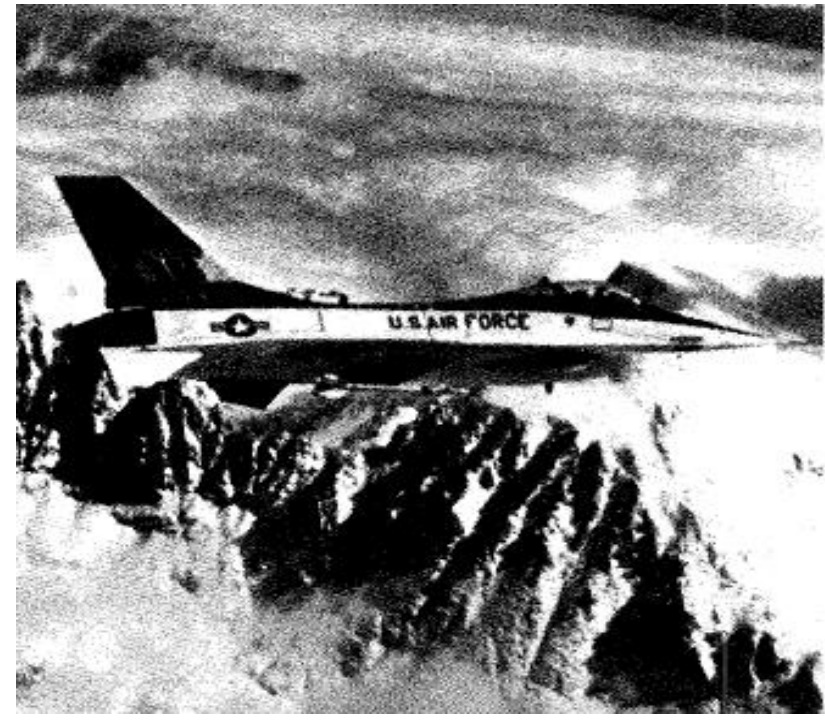

Fig.-12. Output of BBHE

\section{PROPOSED WORK}

In this paper proposes an robust method to enhance the brightness as well as preservation of feature present in an image. To improve the dimmed images via multilevel thresholding, gamma correction and probability based entropy. The enhanced image by these approach Preserve the brightness and feature in the images.

\subsection{Gamma Correction}

Gamma correction can robotically enhance the image contrast. Gamma correction is a non-linear operation ,which is used to correcting lightness or darkness of image[4]. According to the gamma value only image brightness can be corrected. Gamma value ranging from 0.0 to 10.0 . If gamma value less then $1.0(<1.0)$, image gets darken. Else if gamma value greater than $1.0(>1.0)$, image gets lighten. Else gamma value equal to 1 , no changes in an image. Gamma is apply only for display image not to the data of image. Within gamma correction three major process are done. First Histogram analysis, which provides the spatial information of an input image. In the second step, the weighting distribution is used to even the irregular occurrence and thus avoid generation of inauspicious artifacts. In the third and final step, gamma correction can robotically enhance the image.

$\rightarrow$ It defines the association between a pixel's numerical value and its actual brightness.

$\rightarrow$ Study the intensity of the image. Based on the image intensity calculate the gamma value.

$\rightarrow$ Apply the gamma value on the original image to improve the brightness as well Preserve the brightness of the original image.

Gamma parameters calculate as:

$$
\gamma=1-c d f_{\omega}(l)
$$




\subsection{Probability Based Entropy}

The image enhancement is depending on information that present in an image. Based on the including point and excluding point the entropy is computed. The image that is produced by the method is clearly understandable.

$\rightarrow$ With help of using entropy model can calculate information contribution.

$\rightarrow$ Compute the distribution of Normalized numbers of occurrences of each intensity.

Consider the histogram as probability distribution.

$$
p d f(l)=n 1 /(M N)
$$

Number of pixels that have intensity $l, M N$ is the total number of pixels in the image.

$\rightarrow$ The pixels with low information contribution represented by low intensities

$\rightarrow$ The pixels with high information contribution represented by high intensities

$\rightarrow$ Optimize the cross-entropy between the input gray-level image and the output binary image as pinpointing of maintenance of information.

\subsection{Multilevel Thresholding}

Multilevel thresholding produce more than one threshold value for an input image. Convert gray scale image into a binary image [6]. Then calculates the optimum threshold divide those two classes so that they combined spread is least. This approach divide image based on those pixel value are under the threshold it is consider as background value, if not it consider as foreground value.

$\rightarrow$ Multilevel thresholding suitable for either a grayscale or color image as input and, outputs a binary image represent the segmentation. Each pixel in the input image, a threshold has to be calculated.

$\rightarrow$ Those pixel values are under the threshold it is consider as background value, if not it consider as foreground value.

$\rightarrow \mathrm{T}$ the mean of the minimum and maximum values is used to calculate the threshold.

$\rightarrow$ Based on the threshold value image gets energetically change, so image visual quality is improved well.

$\rightarrow$ Visual quality is improved then able to bring out the details or feature hidden in an image.

$\rightarrow$ The enhanced image clear with more details, and visual quality of image improved well.

\section{EXPERIMENTAL RESULTS}

The dimmed and indoor, outdoor images are taken as a input image, which is unable to get the feature from the image. In gamma correction, it defines the association between a pixel's numerical value and its actual brightness. Find out the gamma value and apply that value on original image, which improve the brightness. Probability based entropy for preservation of information and find out low intensity and high intensity pixel. Finally multilevel thresholding, based on threshold value improve the image visual quality. Result of the enhanced image intelligible is measured using in term of entropy value. The resulting of the proposed method preserves the feature of the image and brightness and also improves image quality.

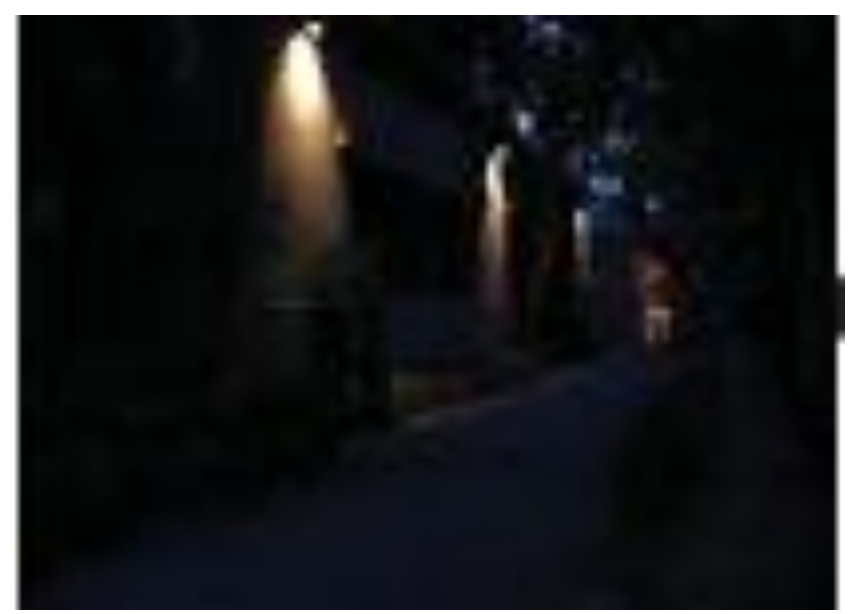

Fig.-13. Original image

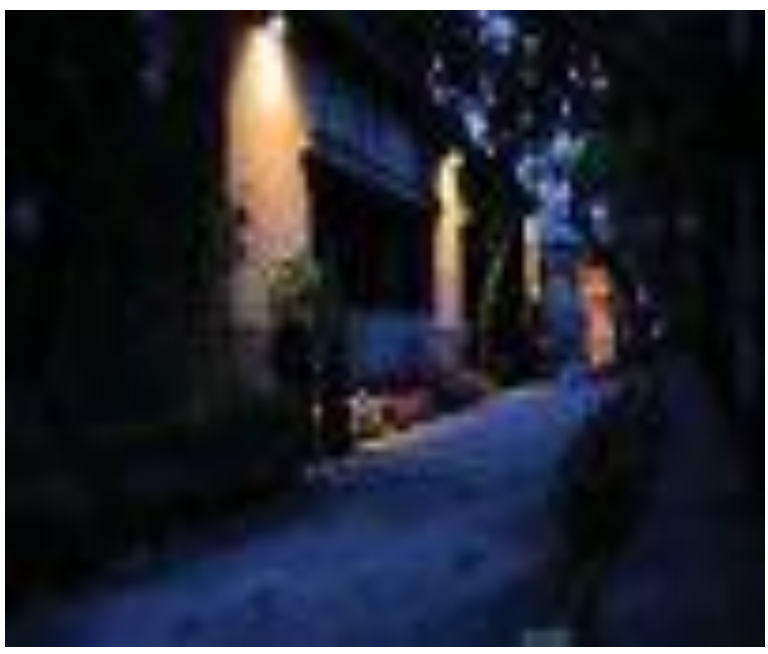

Fig.-14. Enhanced image

\section{CONCLUSIONS}

This paper presents a new enhancement technique for paper improves the brightness of the images and preservation of feature present in an image. In gamma correction, it defines the association between a pixel's numerical value and its actual brightness. Find out the gamma value and apply that value on original image, which improve the brightness. Probability based entropy for preservation of information and find out low intensity and high intensity pixel.

Finally multilevel thresholding, based on threshold value improve the image visual quality. Result of the enhanced image intelligible is measured using in term of entropy value. The enhanced image using the proposed method bring out more details of the original image and visual quality is improved well. 


\section{REFERENCES}

[1]. Rafael C Gonzalez and Richard E Woods, "Digital Image processing", third edition, Pearson Education, 2007.

[2]. J. Alex Stark "Adaptive Image Contrast Enhancement Using Generalizations of Histogram equalization”, IEEE Transactionson Image Processing, Vol. 9, No. 5, May 20

[3]. T. Celik and T. Tjahjadi, "Contextual andvariationalcontrast enhancement,"IEEETrans. Image Process. vol. 20, no. 12, pp. 3431-3441, Dec. 2011

[4]. Y.-S. Chiu, F.-C. Cheng, and S.-C. Huang,"Efficient contrast enhancement Using adaptive gamma correction and cumulative intensity distribution, "in Proc.IEEE Conf. Syst. Man Cybern., Oct. 2011,pp. 2946-2950.

[5]. Chao Wang and Zhongfu Ye "Brightness Preserving Histogram Equalization with Maximum Entropy: A Variational Perspective", Vol. 51, No. 4, November 2005.

[6]. L. Hertz, R.W. Schafer, Multilevel thresholding using edge matching, Computer Vision Graphics and Image Processing 44 (1988) 279-295.

[7]. Y.-T. Kim, "Contrast enhancement using brightness preserving bi-histogram equalization," IEEE Trans. Consumer Electron., vol. 43, no. 1, pp. 1-8, Feb 1997.

[8]. M. Kim and M. G. Chung, "Recursively separated and weighted histogram equalization for brightness preservation and contrast enhancement,"IEEE Trans. Consum. Electron. vol. 54, no. 3, pp.

[9]. D. Menotti, L. Najman, J. Facon, and A. A. Araujo, "Multi-histogram equalization methods for contrast enhancement and brightness preserving," IEEE Transactions on Consumer Electronics, vol. 53, no. 3, pp. 1186-1194, 2007.

[10]. K. S. Sim, C. P. Tso, and Y. Y. Tan, "'Recursive subimage histogram equalization applied to gray scale images"', Pattern Recognition Letters, 28(10), pp. 12091221, 2007.

[11]. Y. Wang, Q. Chen, and B. Zhang, "Image enhancement based on equal area dualistic sub-image histogram equalization method," IEEE Trans. ConsumerElectron., vol. 45, no. 1, pp. 68-75, Feb 1999.

[12]. Q. Wang and R. K. Ward, "Fast image/video contrast enhancement based on weighted thresholded histogram equalization," IEEE Transactions on Consumer Electronics, vol. 53, no. 2, pp. 757-764, 2007.

\section{BIOGRAPHIE}

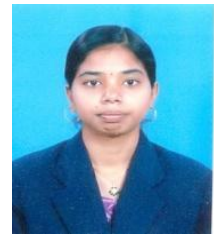

S.Gayathri received B.E degree from Anna University, Chennai (Tamilnadu) in 2012. Currently she is pursuing M.E degree in Computer Science from Anna University, Chennai (Tamilnadu) Her research interest includes Image Processing .She has published papers in International Journals. 